\title{
On Harnack inequalities and optimal transportation
}

\author{
DOMINIQUE BAKRY, IVAN GENTIL AND MiCHEL LEDOUX
}

\begin{abstract}
We develop connections between Harnack inequalities for the heat flow of diffusion operators with curvature bounded from below and optimal transportation. Through heat kernel inequalities, a new isoperimetric-type Harnack inequality is emphasized. Commutation properties between the heat and HopfLax semigroups are developed consequently, providing direct access to heat flow contraction in Wasserstein spaces
\end{abstract}

Mathematics Subject Classification (2010): 35K08 (primary); 60J60, 58J60, 53C21, 46E35, 35B65 (secondary).

\section{Introduction}

Harnack inequalities classically provide strong tools towards regularity properties of solutions of partial differential equations and heat kernel bounds. A renowned result on the topic is the parabolic inequality by P. Li and S.-T. Yau [29]

$$
\frac{\left|\nabla P_{t} f\right|^{2}}{\left(P_{t} f\right)^{2}}-\frac{\Delta P_{t} f}{P_{t} f} \leq \frac{n}{2 t}
$$

for the heat semigroup $\left(P_{t}\right)_{t \geq 0}$ on an $n$-dimensional Riemannian manifold $(M, g)$ with non-negative Ricci curvature, and every $t>0$ and positive (measurable) function $f: M \rightarrow \mathbb{R}$. By integration along geodesics, it yields the Harnack inequality

$$
P_{t} f(x) \leq P_{t+s} f(y)\left(\frac{t+s}{t}\right)^{n / 2} \mathrm{e}^{d(x, y)^{2} / 4 s}
$$

for $f: M \rightarrow \mathbb{R}$ non-negative and $t, s>0$, where $d(x, y)$ is the Riemannian distance between $x, y \in M$. The results (1.1) and (1.2) admit versions for any lower bound on the Ricci curvature ( $c f$. [18,29]). A heat flow proof of $(1.1)$, in the spirit of the arguments developed in this work, has been provided in [10].

In the context of diffusion operators, the Harnack inequality (1.2) may actually loose its relevance due to the infinite-dimensional feature of some models. Let 
$\mathrm{L}=\Delta-\nabla V \cdot \nabla$ be a diffusion operator on a smooth complete connected Riemannian manifold $(M, g)$, where $V: M \rightarrow \mathbb{R}$ is a smooth potential, with associated Markov semigroup $\left(P_{t}\right)_{t \geq 0}$ and invariant and symmetric measure $d \mu=\mathrm{e}^{-V} d x$ (where $d x$ is the Riemannian volume element). A notion of curvature-dimension $C D(K, N), K \in \mathbb{R}, N \geq 1$, of such operators L has been introduced by D. Bakry and M. Émery [7] (cf. $[5,8])$, which is by now classically referred to as the $\Gamma_{2}$ criterion, through the Bochner-type inequality

$$
\frac{1}{2} \mathrm{~L}\left(|\nabla f|^{2}\right)-\nabla f \cdot \nabla \mathrm{L} f \geq K|\nabla f|^{2}+\frac{1}{N}(\mathrm{~L} f)^{2}
$$

for any smooth $f: M \rightarrow \mathbb{R}$. (The $\Gamma_{2}$ operator in this context is precisely the expression on the right-hand side of (1.3).) For example, by the standard Bochner formula from Riemannian geometry, the Laplace operator $\Delta$ on an $n$-dimensional Riemannian manifold with Ricci curvature bounded from below by $K$ satisfies the curvature-dimension condition $C D(K, N)$ with $N \geq n$. On the other hand, on $M=\mathbb{R}^{n}$ with $V$ the quadratic potential, the associated Ornstein-Uhlenbeck operator $\mathrm{L}$ is intrinsically of infinite dimension $N=\infty$ since (1.3) cannot hold for some $K \in \mathbb{R}$ with $N$ finite. (It actually holds in this example with $K=1$ and $N=\infty$.) In particular a Harnack inequality (1.2) cannot hold in this case, as well as in further similar infinite-dimensional models. Note that, when $N=\infty$, again by the Bochner formula, the curvature condition $C D(K, \infty), K \in \mathbb{R}$, amounts to the local geometric lower bound

$$
\operatorname{Ric}+\operatorname{Hess}(V) \geq K
$$

(as symmetric matrices) uniformly over the manifold (cf.e.g. [8]).

To circumvent the drawbacks attached to the case $K=\infty$, F.-Y. Wang introduced in [38] (see also [39]) a new form of Harnack inequalities for infinitedimensional diffusion operators of the type $\mathrm{L}=\Delta-\nabla V \cdot \nabla$ (and more general ones). Wang's Harnack inequalities indicate that, under the curvature condition $C D(K, \infty)$ (equivalent to (1.4)), for every non-negative (Borel measurable) function $f$ on $M$, every $t>0$, every $\alpha>1$, and every $x, y \in M$,

$$
\left(P_{t} f(x)\right)^{\alpha} \leq P_{t}\left(f^{\alpha}\right)(y) \mathrm{e}^{\alpha d(x, y)^{2} / 2(\alpha-1) \sigma(t)}
$$

where $\sigma(t)=\frac{1}{K}\left(\mathrm{e}^{2 K t}-1\right)(=2 t$ if $K=0)$. The proof of (1.5) is based on the interpolation

$$
P_{s}\left(\left(P_{t-s} f\right)^{\alpha}\right)\left(x_{s}\right), \quad s \in[0, t],
$$

along a geodesic $\left(x_{s}\right)_{s \in[0, t]}$ joining $x$ to $y$ together with the commutation, for all $t \geq 0$ and smooth $g: M \rightarrow \mathbb{R}$,

$$
\left|\nabla P_{t} g\right| \leq \mathrm{e}^{-K t} P_{t}(|\nabla g|)
$$

as an equivalent formulation of the curvature lower bound $C D(K, \infty)(c f$. [8]). In a sense, the gradient bound (1.6) may be thought of as the counterpart of the Li-Yau inequality (1.1) in this context. 
Changing $f$ into $f^{1 / \alpha}$, in the asymptotics $f^{1 / \alpha} \sim 1+\frac{1}{\alpha} \log f$ as $\alpha \rightarrow \infty$ in (1.5), a log-Harnack inequality

$$
P_{t}(\log f)(x) \leq \log P_{t} f(y)+\frac{d(x, y)^{2}}{2 \sigma(t)}
$$

also holds $(c f$. $[12,40])$. It was further shown in $[38,40]$ that either (1.5) (for one $\alpha>1$ ) or (1.7) imply back, as $t \rightarrow 0$, the curvature condition $C D(K, \infty)$ (in its infinitesimal form (1.4)).

The aim of this work is two-fold. We will first show how the previous infinitedimensional Harnack inequalities may actually be seen as consequences of a suitable functional inequality of isoperimetric type. On the basis of this observation, we establish next a kind of isoperimetric-type Harnack inequality. These results naturally lead to develop connections between isoperimetric-type Harnack inequalities (in direct or reverse form) and commutation properties between diffusion and Hopf-Lax semigroups. By the dual Kantorovich optimal transportation formalism, Wasserstein contraction properties along the heat flow are then derived.

Two observations are actually at the starting point of this work. For simplicity in the (somewhat informal) discussion below, we restrict ourselves to the curvature condition $C D(0, \infty)$ (with thus $K=0$ ).

First, the gradient bound (1.6) (and thus the curvature condition $C D(0, \infty)$ ) is known to imply (to be equivalent) to logarithmic Sobolev inequalities under the heat kernel measures $P_{t}$, in particular in reverse form

$$
t \frac{\left|\nabla P_{t} f\right|^{2}}{P_{t} f} \leq P_{t}(f \log f)-P_{t} f \log P_{t} f
$$

for every (bounded measurable) $f>0$ and every $t>0(c f$. [8]). Inequalities like the preceding one are understood point-wise throughout this work. Now, as was noticed by M. Hino [24], the latter ensures that whenever $0<f \leq 1$ and $\psi=\sqrt{\log \left(1 / P_{t} f\right)}$, then

$$
|\nabla \psi|^{2} \leq \frac{1}{2 t}
$$

In other words, $\psi$ is Lipschitz with Lipschitz coefficient less than or equal to $(2 t)^{-1 / 2}$. In particular, for every $x, y \in M$,

$$
\sqrt{\log \frac{1}{P_{t} f(x)}} \leq \sqrt{\log \frac{1}{P_{t} f(y)}}+\frac{d(x, y)}{\sqrt{2 t}}
$$

where we recall that $d(x, y)$ is the Riemannian distance between $x$ and $y$. After some work, it may then be shown that for each $\varepsilon>0$, there exists $C(\varepsilon)>0$ such that

$$
\left(P_{t} f(x)\right)^{2} \leq C(\varepsilon) P_{t}\left(f^{2}\right)(y) \mathrm{e}^{d(x, y)^{2} / 2(1+\varepsilon) t},
$$

that is as close as possible to (1.5) (for $\alpha=2$ ). 
It should be mentioned that it is precisely the dimensional version of the reverse logarithmic Sobolev inequality (1.8) which has been used in [10] to provide a monotonicity proof of the Li-Yau parabolic inequality (1.1). We will exploit this information towards dimensional statements in Section 5 below. For further dimensional Harnack inequalities under the curvature-dimension condition $C D(K, N)$, comparing in particular different times, see $[20,27,41]$.

The second observation at the starting point of this investigation is the link between Harnack-type inequalities and optimal transportation already put forward in [12] where semigroup tools were developed towards a proof of the Otto-Villani HWI inequality [32] (cf. [36,37]). We briefly recall the basic step. Namely, the log-Harnack inequality (1.7) may be translated equivalently as

$$
P_{t}(\log f) \leq Q_{2 t}\left(\log P_{t} f\right)
$$

where $\left(Q_{s}\right)_{s>0}$ is the Hopf-Lax infimum-convolution semigroup

$$
Q_{s} \varphi(x)=\inf _{y \in M}\left[\varphi(y)+\frac{d(x, y)^{2}}{2 s}\right], \quad x \in M, s>0 .
$$

Assume now that $\mu$ is a probability measure and let $f>0$ be a (bounded) probability density with respect to $\mu$. Then, by time reversibility and (1.9) applied to $P_{t} f$, $t>0$,

$$
\int_{M} P_{t} f \log P_{t} f d \mu=\int_{M} f P_{t}\left(\log P_{t} f\right) d \mu \leq \int_{M} f Q_{2 t}\left(\log P_{2 t} f\right) d \mu .
$$

Now $\int_{M} \log P_{2 t} f d \mu \leq 0$ by Jensen's inequality. Hence, combining with the scaling properties of $\left(Q_{s}\right)_{s>0}$,

$$
\int_{M} P_{t} f \log P_{t} f d \mu \leq \frac{1}{2 t}\left[\int_{M} Q_{1} \varphi f d \mu-\int_{M} \varphi d \mu\right]
$$

where $\varphi=2 t \log P_{2 t} f$. Recall then the (quadratic) Wasserstein distance $\mathrm{W}_{2}(\nu, \mu)$ between two probability measures $\mu$ and $v$ on $M$ defined by

$$
\mathrm{W}_{2}(\nu, \mu)=\left(\int_{M \times M} d(x, y)^{2} d \pi(x, y)\right)^{1 / 2}
$$

where the infimum is taken over all couplings $\pi$ with respective marginals $v$ and $\mu$. The Kantorovich dual description

$$
\frac{1}{2} \mathrm{~W}_{2}(\nu, \mu)^{2}=\sup \left(\int_{M} Q_{1} \varphi d v-\int_{M} \varphi d \mu\right)
$$

where the supremum runs over all bounded continuous functions $\varphi: M \rightarrow \mathbb{R}$ (cf. e.g. [36]) then yields with $d \nu=f d \mu$

$$
\int_{M} P_{t} f \log P_{t} f d \mu \leq \frac{1}{4 t} \mathrm{~W}_{2}^{2}(\nu, \mu) .
$$


Note that the preceding argument similarly yields, for every $t>0$,

$$
\int_{M} P_{t} f \log P_{t} f d \mu \leq \frac{1}{4 t} \mathrm{~W}_{2}^{2}(f \mu, g \mu)+\int_{M} g \log g d \mu
$$

where $g$ is a further probability density with respect to $\mu$, and where, for simplicity here, $f \mu$ and $g \mu$ denote the probability measures $f d \mu$ and $g d \mu$. Indeed, write in the preceding notation that

$$
\int_{M} P_{t} f \log P_{t} f d \mu \leq \frac{1}{2 t}\left[\int_{M} Q_{1} \varphi f d \mu-\int_{M} \varphi g d \mu\right]+\int_{M} g \log P_{2 t} f d \mu .
$$

Since by convexity $\int_{M} g \log P_{2 t} f d \mu \leq \int_{M} g \log g d \mu$, the claim follows.

Now (1.11) is actually the major step in the semigroup proof of the HWI inequality of [32] under the curvature condition $C D(0, \infty)$. Namely, the classical heat flow interpolation scheme $(c f .[5,8])$ indicates that, for every suitable smooth probability density $f: M \rightarrow \mathbb{R}$ and every $t \geq 0$,

$$
\begin{aligned}
\int_{M} f \log f d \mu-\int_{M} P_{t} f \log P_{t} f d \mu & =-\int_{0}^{t}\left(\frac{d}{d s} \int_{M} P_{s} f \log P_{s} f d \mu\right) d s \\
& =\int_{0}^{t} \int_{M} \frac{\left|\nabla P_{s} f\right|^{2}}{P_{s} f} d \mu d s .
\end{aligned}
$$

Since $\left|\nabla P_{S} f\right| \leq P_{S}(|\nabla f|)$ according to (1.6),

$$
\frac{\left|\nabla P_{s} f\right|^{2}}{P_{s} f} \leq P_{s}\left(\frac{|\nabla f|^{2}}{f}\right)
$$

by the Cauchy-Schwarz inequality along the Markov kernel $P_{s}$. Therefore,

$$
\int_{M} f \log f d \mu \leq \int_{M} P_{t} f \log P_{t} f d \mu+t \int_{M} \frac{|\nabla f|^{2}}{f} d \mu .
$$

Together thus with (1.11), optimization in $t>0$ yields

$$
\int_{M} f \log f d \mu \leq \mathrm{W}_{2}(\nu, \mu)\left(\int_{M} \frac{|\nabla f|^{2}}{f} d \mu\right)^{1 / 2}
$$

which is the announced HWI inequality, connecting Entropy, Wasserstein distance and Fisher Information. Similar arguments may be developed under $C D(K, \infty)$ for any $K \in \mathbb{R}$ to yield the full formulation of Otto-Villani's HWI inequality $(c f .[8,12])$. Note that together with $(1.11)$, the argument also recovers the known inequality (cf.e.g. [16])

$$
\int_{M} f \log f d \mu \leq \mathrm{W}_{2}(f \mu, g \mu)\left(\int_{M} \frac{|\nabla f|^{2}}{f} d \mu\right)^{1 / 2}+\int_{M} g \log g d \mu
$$

for probability densities $f$ and $g$ with respect to $\mu$. 
For the matter of comparison, it might be worthwhile mentioning that the recent Kuwada lemma (see [22]) develops similar arguments towards the inequality

$$
W_{2}^{2}\left(P_{t} f \mu, f \mu\right) \leq t\left[\int_{E} f \log f d \mu-\int_{E} P_{t} f \log P_{t} f d \mu\right]
$$

for any probability density $f$ with respect to $\mu$ and any $t \geq 0$. Indeed, for $\varphi: E \rightarrow \mathbb{R}$ bounded and continuous,

$$
\begin{aligned}
\int_{M} Q_{1} \varphi P_{t} f d \mu-\int_{M} \varphi f d \mu & =\int_{0}^{1}\left(\frac{d}{d s} \int_{M} Q_{s} \varphi P_{s t} f d \mu\right) d s \\
& =\int_{0}^{1} \int_{M}\left[-\frac{1}{2}\left|\nabla Q_{s} \varphi\right|^{2} P_{s t} f+t Q_{s} \varphi \mathrm{L} P_{t s} f\right] d \mu d s \\
& =\int_{0}^{1} \int_{M}\left[-\frac{1}{2}\left|\nabla Q_{s} \varphi\right|^{2} P_{s t} f-t \nabla Q_{s} \varphi \cdot \nabla P_{s t} f\right] d \mu d s
\end{aligned}
$$

by the fact that the Hopf-Lax semigroup solves the standard Hamilton-Jacobi equation and by integration by parts. Next, by the Cauchy-Schwarz inequality,

$$
\int_{M} Q_{1} \varphi P_{t} f d \mu-\int_{M} \varphi f d \mu \leq \frac{t^{2}}{2} \int_{0}^{1} \int_{M} \frac{\left|\nabla P_{s t} f\right|^{2}}{P_{s t} f} d \mu d s
$$

which yields (1.13) by the Kantorovich duality and integration.

The inequality (1.13) is actually at the core of the gradient flow interpretation of the heat flow in Wasserstein space $(c f .[1,25,31,36,37])$, and immediately follows for example from the Benamou-Brenier [11] dynamical characterization of the Wasserstein distance in smooth spaces such as Riemannian manifolds. Kuwada's argument above extends its validity to a general, possibly nonlinear, setting.

From the point of view of functional inequalities, (1.13) somewhat works in the other direction with respect to (1.11). Namely, while (1.11) leads to the HWI inequality, (1.13) has been identified in [23] at the root of the Otto-Villani theorem [32] (cf. $[12,36,37])$ connecting logarithmic Sobolev inequalities to transportation cost inequalities.

On the basis of these two early observations, the purpose of this work is, as announced, to develop a synthetic and refined treatment of Harnack-type inequalities for diffusion operators with curvature bounded from below and of their connections with transportation cost inequalities. The various contributions of this work are summarized as follows.

In Section 2, we provide a direct treatment of Wang's Harnack inequalities (1.5) and (1.7) relying on an improved, isoperimetric-type version, of the reverse logarithmic Sobolev inequality (1.8) along the heat flow.

This reverse isoperimetric-type inequality in turn implies a new isoperimetric version of Harnack inequalities emphasized in Section 3. For example, under nonnegative curvature $C D(0, \infty)$, it yields that for any (Borel) measurable set $A$ in $M$, 
any $t>0$ and any $x, y \in M$,

$$
P_{t}\left(\mathbf{1}_{A}\right)(x) \leq P_{t}\left(\mathbf{1}_{A_{d(x, y)}}\right)(y)
$$

where $A_{\varepsilon}$ is the $\varepsilon$-neighborhood of $A$ in the metric $d$. This result seems to be new even for the standard heat flow operator on a Riemannian manifold. It is optimal for the standard heat kernel on $\mathbb{R}^{n}$ as is immediately checked on the explicit kernel representation.

A direct consequence of (1) is the commutation

$$
P_{t}\left(Q_{s}\right) \leq Q_{s}\left(P_{t}\right), \quad t, s>0
$$

between the heat and Hopf-Lax semigroups (under non-negative curvature) which we emphasize in Section 4. This commutation was actually used earlier by K. Kuwada [26] in the study of the duality of gradient estimates at the root of the contraction property of the Wasserstein distance along the heat flow

$$
\mathrm{W}_{2}\left(\mu_{t}, v_{t}\right) \leq \mathrm{W}_{2}\left(\mu_{0}, v_{0}\right)
$$

where $d \mu_{t}=P_{t} f d \mu$ and $d v_{t}=P_{t} g d \mu, t \geq 0, f, g$ probability densities with respect to $\mu$. Such contraction properties have been investigated in this context in $[15,31,33,34]$ (see also $[36,37,39]$ ), and are also, following $[1,19]$, a main consequence of the EVI approach discussed in Section 6.

The commutation property (1.14) may actually be reached in several ways, and Section 5 presents a variety of methods depending on the underlying context, including the original approach of [26]. This section further includes dimensional versions of the commutation property together with the corresponding Wassertein contractions.

In the last Section 6, we briefly discuss some connections between the material presented here and recent developments, following [4], around the Evolutionary Variational Inequality (EVI) expressing in the preceding notation that

$$
\mathrm{W}_{2}^{2}\left(\mu_{t}, v_{0}\right)+2 t \int_{M} P_{t} f \log P_{t} f d \mu \leq \mathrm{W}_{2}^{2}\left(\mu_{0}, \nu_{0}\right)+2 t \int_{M} g \log g d \mu .
$$

This property actually connects the $\Gamma_{2}$ Bakry-Émery $C D(K, \infty)$ curvature condition $[5,7,8]$, expressed by the commutation (1.6), with the curvature bound in the sense of Lott-Villani-Sturm in metric measure spaces as convexity of relative entropy along the geodesics of optimal transportation $[30,35,37]$. The recent main achievement by L. Ambrosio, N. Gigli and G. Savaré [2-4] actually provides a link between the $\Gamma_{2}$ and Lott-Villani-Sturm curvature lower bounds in the class of the Riemannian energy measure spaces through the EVI. In Section 6, we sketch, following [4], the principle of proof of the EVI in a smooth setting, for comparison with some of the tools developed here.

For simplicity in the exposition, the results of this work are presented in the weighted Riemannian setting, for thus diffusion operators $\mathrm{L}=\Delta-\nabla \cdot \nabla V$ on 
a complete connected Riemannian manifold $(M, g)$ with invariant and reversible measure $d \mu=\mathrm{e}^{-V} d x$ (not necessarily a probability measure) where $V: M \rightarrow \mathbb{R}$ is a smooth potential. Integration by parts with respect to $\mathrm{L}$ is expressed by $\int_{M} f(-\mathrm{L} g) d \mu=\int_{M} \nabla f \cdot \nabla g d \mu$ for smooth functions $f, g: M \rightarrow \mathbb{R}$. The associated curvature condition $C D(K, \infty), K \in \mathbb{R}$, is expressed equivalently by (1.4) as the infinitesimal version of the Bochner-type inequality (1.3). It amounts to the standard lower bound on the Ricci curvature for the Laplace operator $\Delta$ on $(M, g)$. The curvature condition $C D(K, \infty)$ is also equivalent to the gradient bound (1.6) which is, in an essential manner, the only way the curvature condition will be used throughout this work.

We refer to the general references $[5,8]$ for a precise description of this framework and the relevant properties. Most of the results below actually extend to the more general setting of a Markov diffusion Triple $(E, \mu, \Gamma)$ emphasized in $[5,8]$, consisting of a state space $E$ equipped with a diffusion semigroup $\left(P_{t}\right)_{t>0}$ with infinitesimal generator $\mathrm{L}$ and carré du champ operator $\Gamma$ and invariant and reversible $\sigma$-finite measure $\mu$. In the weighted Riemannian context, $\Gamma(f, f)=|\nabla f|^{2}$ for smooth functions. In this setting, the abstract curvature condition $C D(K, \infty)$, $K \in \mathbb{R}$, stems from the Bochner-type inequality (1.3) (with $N=\infty$ ) and the abstract $\Gamma_{2}$ operator going back to [7] (see [5,8]). The condition $C D(K, \infty)$ is equivalent to the gradient bound (1.6)

$$
\sqrt{\Gamma\left(P_{t} f\right)} \leq \mathrm{e}^{-K t} P_{t}(\sqrt{\Gamma(f)})
$$

for every $t \geq 0$ and every $f$ in a suitable algebra of functions. The state space $E$ may be endowed with an intrinsic distance $d$ for which Lipschitz functions $f$ are such that $\Gamma(f)$ is bounded ( $\mu$-almost everywhere). Note also that at the level of the local inequalities along the semigroup, generators of the type $\mathrm{L}=\Delta+Z$ for some smooth vector field $Z$ on a manifold $M$ may be covered similarly as developed in $[38-40]$.

ACKnowledgements. We are thankful to L. Ambrosio, N. Gigli and G. Savaré for helpful discussions on the EVI and for pointing out relevant references, and to A. Guillin for sharing with us his simple proof from the Wasserstein contraction to the curvature condition at the end of Section 4. We are also most grateful to the referee for numerous comments and corrections that helped in improving the exposition.

\section{Reverse isoperimetry and Wang's Harnack inequalities}

In this section, we address a direct proof of Wang's Harnack inequalities (1.5) and (1.7) along the Hino argument on the basis of a reinforced family of heat kernel inequalities first emphasized in [9]. 
Denote by $I:[0,1] \rightarrow \mathbb{R}_{+}$the Gaussian isoperimetric function defined by $I=\varphi \circ \Phi^{-1}$ where

$$
\Phi(x)=\int_{-\infty}^{x} \mathrm{e}^{-u^{2} / 2} \frac{d u}{\sqrt{2 \pi}}, \quad x \in \mathbb{R}
$$

and $\varphi=\Phi^{\prime}$. The function $I$ is concave continuous, symmetric with respect to the vertical line going through $\frac{1}{2}$ and such that $I(0)=I(1)=0$, and satisfies the basic differential equality $I I^{\prime \prime}=-1$. Moreover $I(v) \sim v \sqrt{2 \log \frac{1}{v}}$ as $v \rightarrow 0$.

The following statement, as a kind of reverse isoperimetric-type inequality, was first put forward in [9]. We enclose a proof for completeness (see also [8]).

Proposition 2.1. Under the curvature condition $C D(K, \infty)$ for some $K \in \mathbb{R}$, for every (measurable) function $f$ on $M$ with values in $[0,1]$ and every $t>0$,

$$
\left[I\left(P_{t} f\right)\right]^{2}-\left[P_{t}(I(f))\right]^{2} \geq \sigma(t)\left|\nabla P_{t} f\right|^{2}
$$

where $\sigma(t)=\frac{1}{K}\left(\mathrm{e}^{2 K t}-1\right)(=2 t$ if $K=0)$.

Proof. Work with a function $f$ such that $\varepsilon \leq f \leq 1-\varepsilon$ for some $\varepsilon>0$. By the heat flow interpolation, write

$$
\left[I\left(P_{t} f\right)\right]^{2}-\left[P_{t}(I(f))\right]^{2}=-\int_{0}^{t} \frac{d}{d s}\left[P_{s}\left(I\left(P_{t-s} f\right)\right)\right]^{2} d s .
$$

Now, by the chain rule for the diffusion operator L,

$$
\begin{aligned}
-\frac{d}{d s}\left[P_{s}\left(I\left(P_{t-s} f\right)\right)\right]^{2} & \left.=-2 P_{s}\left(I\left(P_{t-s} f\right)\right) P_{s}\left(\mathrm{~L} I\left(P_{t-s} f\right)-I^{\prime}\left(P_{t-s} f\right) \mathrm{L} P_{t-s} f\right)\right) \\
& =-2 P_{s}\left(I\left(P_{t-s} f\right)\right) P_{s}\left(I^{\prime \prime}\left(P_{t-s} f\right)\left|\nabla P_{t-s} f\right|^{2}\right) \\
& =2 P_{s}\left(I\left(P_{t-s} f\right)\right) P_{s}\left(\frac{\left|\nabla P_{t-s} f\right|^{2}}{I\left(P_{t-s} f\right)}\right)
\end{aligned}
$$

where we used that $I I^{\prime \prime}=-1$ in the last step. Since $P_{S}$ is given by a kernel, it satisfies a Cauchy-Schwarz inequality, and hence

$$
P_{S}(Y) P_{s}\left(\frac{X^{2}}{Y}\right) \geq\left[P_{S}(X)\right]^{2}, \quad X, Y \geq 0 .
$$

Hence, with $X=\left|\nabla P_{t-s} f\right|$ and $Y=I\left(P_{t-s} f\right)$,

$$
\left[I\left(P_{t} f\right)\right]^{2}-\left[P_{t}(I(f))\right]^{2} \geq 2 \int_{0}^{t}\left[P_{s}\left(\left|\nabla P_{t-s} f\right|\right)\right]^{2} d s
$$


By the gradient bound (1.6) applied to $g=P_{t-s} f$, it follows that

$$
\left[I\left(P_{t} f\right)\right]^{2}-\left[P_{t}(I(f))\right]^{2} \geq 2 \int_{0}^{t} \mathrm{e}^{2 K s} d s\left|\nabla P_{t} f\right|^{2}
$$

which is the result.

For the comparison with the Hino observation mentioned in the introduction, note that (2.1) of Proposition 2.1 implies the reverse logarithmic Sobolev inequality (1.8) by applying it to $\varepsilon f$ and letting $\varepsilon \rightarrow 0$.

As announced, we next show how Proposition 2.1 actually covers Wang's Harnack inequalities recalled in the introduction. The main consequence is put forward in the following corollary that actually entails most of the inequalities emphasized in this work.

Corollary 2.2. Under the curvature condition $C D(K, \infty)$ for some $K \in \mathbb{R}$, for every (measurable) function $f$ on $M$ with values in $[0,1]$, every $t>0$ and every $x, y \in M$,

$$
\Phi^{-1} \circ P_{t} f(x) \leq \Phi^{-1} \circ P_{t} f(y)+\frac{d(x, y)}{\sqrt{\sigma(t)}}
$$

where $d(x, y)$ the Riemannian distance between $x$ and $y$.

Namely, in terms of gradient bounds, Proposition 2.1 implies that for every $f$ with values in $[0,1]$,

$$
\left|\nabla P_{t} f\right| \leq \frac{1}{\sqrt{\sigma(t)}} I\left(P_{t} f\right)
$$

Since $\left(\Phi^{-1}\right)^{\prime}=\frac{1}{I}$, it follows that $\Phi^{-1} \circ P_{t} f$ is $\sigma(t)^{-1 / 2}$-Lipschitz, $t>0$, which amounts to the corollary.

Towards a first illustration of Corollary 2.2, set $\delta=d(x, y) / \sqrt{\sigma(t)}$, so that (2.2) reads as

$$
P_{t} f(x) \leq \Phi\left(\Phi^{-1} \circ P_{t} f(y)+\delta\right) .
$$

Apply now this inequality to $\mathbf{1}_{\{f \geq a\}}, a \geq 0$, for a non-negative function $f$ on $M$. Denoting by $\lambda$ the distribution of $f$ under $P_{t}$ at the point $y$ (that is $\lambda(B)=$ $P_{t}\left(\mathbf{1}_{\{f \in B\}}\right)(y)$ for every Borel set $B$ in $\left.\mathbb{R}\right)$,

$$
P_{t}\left(\mathbf{1}_{\{f \geq a\}}\right)(x) \leq \Phi\left(\Phi^{-1}(\lambda([a, \infty)))+\delta\right)
$$

Integrating in $a \geq 0$ and using Fubini's theorem, denoting by $d \gamma(u)=\mathrm{e}^{-u^{2} / 2} \frac{d u}{\sqrt{2 \pi}}$ the standard Gaussian distribution on the line,

$$
P_{t} f(x) \leq \int_{0}^{\infty} \int_{-\infty}^{\Phi^{-1}(\lambda([a, \infty)))+\delta} d \gamma(u) d a=\int_{-\infty}^{\infty}\left(\int_{0}^{\infty} \mathbf{1}_{\left\{u \leq \Phi^{-1}(\lambda([a, \infty)))+\delta\right\}} d a\right) d \gamma(u) .
$$


Change $u$ into $u+\delta$ to get

$$
P_{t} f(x) \leq \mathrm{e}^{-\delta^{2} / 2} \int_{-\infty}^{\infty} \mathrm{e}^{-\delta u}\left(\int_{0}^{\infty} \mathbf{1}_{\{\Phi(u) \leq \lambda([a, \infty))\}} d a\right) d \gamma(u) .
$$

Changing $u$ into $-u$ and denoting by $F$ the distribution function of $\lambda$, it follows that

$$
P_{t} f(x) \leq \mathrm{e}^{-\delta^{2} / 2} \int_{-\infty}^{\infty} \mathrm{e}^{\delta u}\left(\int_{0}^{\infty} \mathbf{1}_{\{F(a) \leq \Phi(u)\}} d a\right) d \gamma(u) .
$$

After the further change of variables $v=\Phi(u)$,

$$
P_{t} f(x) \leq \mathrm{e}^{-\delta^{2} / 2} \int_{0}^{1} \mathrm{e}^{\delta \Phi^{-1}(v)}\left(\int_{0}^{\infty} \mathbf{1}_{\{F(a) \leq v\}} d a\right) d v .
$$

The next statement summarizes the conclusion reached so far.

Theorem 2.3. Under the curvature condition $C D(K, \infty)$ for some $K \in \mathbb{R}$, for every non-negative (measurable) function $f$ on $M$, every $t>0$ and every $x, y \in M$,

$$
P_{t} f(x) \leq \mathrm{e}^{-\delta^{2} / 2} \int_{0}^{\infty} \mathrm{e}^{\delta \Phi^{-1} \circ F(r)} r d F(r)
$$

where $\delta=d(x, y) / \sqrt{\sigma(t)}$ and $F(r)=P_{t}\left(\mathbf{1}_{\{f \leq r\}}\right)(y), r \geq 0$, is the distribution function of $f$ under $P_{t}$ at the point $y$.

Theorem 2.3 appears at the root of the various Harnack inequalities in this context. It is however not expressed in a very tractable form. But it easily implies known ones. For example, by Cauchy-Schwarz,

$$
\begin{aligned}
\int_{0}^{\infty} \mathrm{e}^{\delta \Phi^{-1} \circ F(r)} r d F(r) & \leq\left(\int_{0}^{\infty} \mathrm{e}^{2 \delta \Phi^{-1} \circ F(r)} d F(r)\right)^{1 / 2}\left(\int_{0}^{\infty} r^{2} d F(r)\right)^{1 / 2} \\
& \leq \mathrm{e}^{\delta^{2}}\left(P_{t}\left(f^{2}\right)(y)\right)^{1 / 2}
\end{aligned}
$$

since

$$
\int_{0}^{\infty} \mathrm{e}^{2 \delta \Phi^{-1} \circ F(r)} d F(r)=\int_{0}^{1} \mathrm{e}^{2 \delta \Phi^{-1}(v)} d v=\int_{-\infty}^{\infty} \mathrm{e}^{2 \delta u} d \gamma(u)=\mathrm{e}^{2 \delta^{2}} .
$$

The preceding therefore yields Wang's Harnack inequality (1.5) for $\alpha=2$,

$$
P_{t} f(x)^{2} \leq P_{t}\left(f^{2}\right)(y) \mathrm{e}^{d(x, y)^{2} / \sigma(t)} .
$$

By Hölder's inequality rather than Cauchy-Schwarz, one obtains the whole family of inequalities (1.5) with $\alpha>1$. Using the entropic inequality yields similarly the $\log$-Harnack inequality (1.7). With respect to Wang's original argument, the proof here avoids interpolation along geodesics (although the length space property is required to move from (2.1) to (2.2)). 


\section{Isoperimetric-type Harnack inequalities}

As announced, the basic Lipschitz inequality (2.2) of Corollary 2.2 may be seen at the origin of a number of inequalities of interest, and this section develops further consequences in combination with isoperimetric bounds for heat kernel measures. To this task, recall first the isoperimetric comparison theorem for heat kernel measures under curvature bounds of [9]. Recall $I$ the Gaussian isoperimetric function.

Theorem 3.1. Under the curvature condition $C D(K, \infty)$ for some $K \in \mathbb{R}$, for every smooth function $f$ on $M$ with values in $[0,1]$ and every $t \geq 0$,

$$
I\left(P_{t} f\right) \leq P_{t}\left(\sqrt{I^{2}(f)+K(t)|\nabla f|^{2}}\right)
$$

where $K(t)=\frac{1}{K}\left(1-\mathrm{e}^{-2 K t}\right)(=2 t$ if $K=0)$.

As developed in [9] ( $c f$. also [8]), this result is an isoperimetric comparison theorem expressing that the isoperimetric profile of the heat kernel measures is bounded from below by the Gaussian isoperimetric function (up to a scaling depending on $t$ and $K)$. This comparison may classically $(c f .[9,13])$ be translated in terms of isoperimetric neighborhoods in the sense that for any Borel measurable (or closed) set $A \subset M$ and any $\varepsilon>0$,

$$
P_{t}\left(\mathbf{1}_{A_{\varepsilon}}\right)(y) \geq \Phi\left(\Phi^{-1}\left(P_{t}\left(\mathbf{1}_{A}\right)(y)\right)+\frac{\varepsilon}{\sqrt{K(t)}}\right)
$$

where $A_{\varepsilon}$ is the (closed) $\varepsilon$-neighborhood of $A$ in the distance $d$, for any $y \in M$ and $t>0$.

Applied to $f=\mathbf{1}_{A}$, the Lipschitz property (2.2) ensures on the other hand that, for any measurable set $A \subset M$, and again with $\delta=d(x, y) / \sqrt{\sigma(t)}$,

$$
P_{t}\left(\mathbf{1}_{A}\right)(x) \leq \Phi\left(\Phi^{-1}\left(P_{t}\left(\mathbf{1}_{A}\right)(y)\right)+\delta\right)
$$

The combination of (3.1) and (3.2) together with the fact that $\frac{K(t)}{\sigma(t)}=\mathrm{e}^{-2 K t}$ then yields the following isoperimetric-type Harnack inequality.

Theorem 3.2. Under the curvature condition $C D(K, \infty)$ for some $K \in \mathbb{R}$, for every measurable set $A$ in $M$, every $t \geq 0$ and every $x, y \in M$ such that $d(x, y)>0$,

$$
P_{t}\left(\mathbf{1}_{A}\right)(x) \leq P_{t}\left(\mathbf{1}_{A_{d_{t}}}\right)(y)
$$

where $d_{t}=\mathrm{e}^{-K t} d(x, y)$. In particular, when $K=0$,

$$
P_{t}\left(\mathbf{1}_{A}\right)(x) \leq P_{t}\left(\mathbf{1}_{A_{d(x, y)}}\right)(y) .
$$




\section{The commutation property and contraction in Wasserstein distance}

The isoperimetric-type Harnack inequality of Theorem 3.2 has several consequences of interest in terms of commutation properties between the heat and the HopfLax semigroups which in turn entails the contraction property of the heat flow with respect to Wasserstein metrics.

Recall the Hopf-Lax infimum-convolution semigroup ( $c f .[19,36,37])$

$$
Q_{s} f(x)=\inf _{y \in M}\left[f(y)+\frac{d(x, y)^{2}}{2 s}\right], \quad x \in M, s>0 .
$$

The announced commutation property was actually used first by K. Kuwada [26] in the analysis of gradient bounds and Wasserstein contractions (see Corollary 4.2 below). The proof in [26], developed in the context of length spaces and actually for more general costs, relies on an interpolation along geodesics and the use of the Hamilton-Jacobi equation (see the first alternate proof in Section 5 below).

Theorem 4.1. Under the curvature condition $C D(K, \infty)$ for some $K \in \mathbb{R}$, for any $t, s>0$ and any bounded continuous function $f: M \rightarrow \mathbb{R}$,

$$
P_{t}\left(Q_{s} f\right) \leq Q_{\mathrm{e}^{2 K t_{s}}}\left(P_{t} f\right)
$$

Proof. Let without loss of generality $f$ be non-negative on $M$. It is enough by homogeneity to consider $s=1$. Let $x, y$ be arbitrary (distinct) fixed points in $M$ and set $d_{t}=\mathrm{e}^{-K t} d(x, y)>0$. Set $A=\left\{Q_{1} f \geq a\right\}$ for $a \geq 0$. If $z \in A_{d_{t}}$, there exists $\xi \in A$ such that $d(z, \xi) \leq d_{t}$ so that

$$
f(z)+\frac{d_{t}^{2}}{2} \geq f(z)+\frac{d(z, \xi)^{2}}{2} \geq Q_{1} f(\xi) \geq a .
$$

Hence $A_{d_{t}} \subset\left\{f+d_{t}^{2} / 2 \geq a\right\}$. Therefore, by Theorem 3.2,

$$
P_{t}\left(\mathbf{1}_{\left\{Q_{1} f \geq a\right\}}\right)(x) \leq P_{t}\left(\mathbf{1}_{\left\{f+d_{t}^{2} / 2 \geq a\right\}}\right)(y) .
$$

Integrating in $a \geq 0$ yields

$$
P_{t}\left(Q_{1} f\right)(x) \leq P_{t} f(y)+\frac{d_{t}^{2}}{2}
$$

Taking then the infimum in $y \in M$ yields the result by definition of the infimumconvolution $Q_{1}$.

The infimum-convolution semigroup $\left(Q_{s}\right)_{s>0}$ being solution of the HamiltonJacobi equation $\partial_{s} u=-\frac{1}{2}|\nabla u|^{2}$ with initial condition $u(0, \cdot)=f$, the commutation property (4.1) implies by a Taylor expansion at $s=0$ that $\left|\nabla P_{t} f\right|^{2} \leq \mathrm{e}^{-2 K t} P_{t}\left(|\nabla f|^{2}\right)$ 
for every $t \geq 0$. This gradient bound, weaker than (1.6), is still equivalent to the curvature bound $C D(K, \infty)$ ( $c f$. [8]), providing therefore a converse to Theorem 4.1. In particular also, the isoperimetric Harnack inequality from Theorem 3.2 is actually equivalent to the curvature condition $C D(K, \infty)$.

As announced, it immediately follows from the commutation property (4.1) of Theorem 4.1 that the Wasserstein distance $W_{2}$ is contractive along the semigroup $\left(P_{t}\right)_{t \geq 0}$, an observation due to K. Kuwada [26]. The Wasserstein contraction property in this context may be traced back in the investigation [31] of the heat flow as a gradient flow in the Wasserstein space, further developed in [15]. Further contributions include [34] with a stochastic proof, [33] with an Eulerian point of view, or $[1,19]$ in connection with the EVI (Section 6). See also $[36,37]$. The proof presented here on the basis of Theorem 4.1 extends to the abstract Markov semigroup setting of $[5,8]$. The measure $\mu$ is assumed here to be a probability measure.

Corollary 4.2. Under the curvature condition $C D(K, \infty)$ for some $K \in \mathbb{R}$, for any $t \geq 0$,

$$
W_{2}\left(\mu_{t}, v_{t}\right) \leq \mathrm{e}^{-2 K t} W_{2}\left(\mu_{0}, v_{0}\right)
$$

where $d \mu_{t}=P_{t} f d \mu$ and $d v_{t}=P_{t} g d \mu$ for probability densities $f, g$ with respect to the probability measure $\mu$.

Proof. For any bounded continuous $\varphi: M \rightarrow \mathbb{R}$, by time reversibility and the commutation property (4.1),

$$
\begin{aligned}
\int_{M} Q_{1} \varphi P_{t} f d \mu-\int_{M} \varphi P_{t} g d \mu & =\int_{M} P_{t}\left(Q_{1} \varphi\right) f d \mu-\int_{M} P_{t} \varphi g d \mu \\
& \leq \int_{M} Q_{\mathrm{e}^{2 K t}}\left(P_{t} \varphi\right) f d \mu-\int_{M} P_{t} \varphi g d \mu \\
& \leq \mathrm{e}^{-2 K t}\left[\int_{M} Q_{1}\left(\mathrm{e}^{2 K t} P_{t} \varphi\right) f d \mu-\int_{M} \mathrm{e}^{2 K t} P_{t} \varphi g d \mu\right] \\
& \leq \frac{\mathrm{e}^{-2 K t}}{2} W_{2}^{2}\left(\mu_{0}, v_{0}\right)
\end{aligned}
$$

where the last step follows from the Kantorovich dual description (1.10) of the Wasserstein distance $W_{2}$. The proof is complete.

By adapting Theorem 4.1 to costs $d(x, y)^{p}$, the same argument works for any Wasserstein distance $W_{p}, 1 \leq p<\infty$, extending the contraction property of Corollary 4.2 to this class. More general Wasserstein functionals associated to further transportation costs may be considered similarly. In [26], K. Kuwada established the equivalence of the Wasserstein contraction property for the cost $d(x, y)^{p}$ with the bound (1.6) with power $q$ on the gradient, where $q>1$ and $p<\infty$ are dual exponents.

Note that one main conclusion of the work [34] by M.-K. von Renesse and K.-T. Sturm is the equivalence of the Wasserstein contraction of Corollary 4.2 with 
the curvature bound. Actually, reading backwards the proof of Corollary 4.2, the contraction property (4.2) indicates that for all $\varphi: M \rightarrow \mathbb{R}$ bounded and continuous,

$\int_{M} P_{t}\left(Q_{1} \varphi\right) f d \mu-\int_{M} P_{t} \varphi g d \mu \leq \int_{M} Q_{1} \varphi P_{t} f d \mu-\int_{M} \varphi P_{t} g d \mu \leq \frac{\mathrm{e}^{-2 K t}}{2} W_{2}^{2}\left(\mu_{0}, \nu_{0}\right)$.

Now, if $x, y \in M$ and if $f$ and $g$ are densities with respect to $\mu$ such that $d \mu_{0}=f d \mu$ and $d v_{0}=g d \mu$ approach Dirac masses at $x$ and $y$ respectively (for example by heat kernel approximations), the preceding yields

$$
P_{t}\left(Q_{1} \varphi\right)(x)-P_{t} \varphi(y) \leq \frac{\mathrm{e}^{-2 K t}}{2} d(x, y)^{2},
$$

that is exactly the commutation property (4.1). As we have seen, the latter ensures in turn the curvature condition $C D(K, \infty)$.

\section{Alternate proofs of the commutation property}

In this section, we briefly outline alternate proofs of the basic commutation property (4.1) of Theorem 4.1. For simplicity in the notation and the exposition, we only consider $K=0$ below. Each proof involves at some point specific properties and may thus be adapted to more general settings accordingly.

(i) First alternate proof. This proof is the original argument by K. Kuwada [26]. It requires the use of geodesics and the Hopf-Lax formula as solution of the HamiltonJacobi equation. Consider, for a smooth enough function $f: M \rightarrow \mathbb{R}$,

$$
\phi(s)=P_{t}\left(Q_{s} f\right)\left(x_{s}\right), \quad s \in[0,1],
$$

where $\left(x_{s}\right)_{s \in[0,1]}$ is a constant speed curve joining $x_{0}=y$ to $x_{1}=x$ in $M$. Set for simplicity $d=d(x, y)$. Then, by the gradient bound (1.6) under $C D(0, \infty)$,

$$
\begin{aligned}
\phi^{\prime}(s) & =P_{t}\left(-\frac{1}{2}\left|\nabla Q_{s} f\right|^{2}\right)\left(x_{s}\right)+\nabla P_{t}\left(Q_{s} f\right)\left(x_{s}\right) \cdot \dot{x}_{s} \\
& \leq P_{t}\left(-\frac{1}{2}\left|\nabla Q_{s} f\right|^{2}\right)\left(x_{s}\right)+d\left|\nabla P_{t}\left(Q_{s} f\right)\left(x_{s}\right)\right| \\
& \leq P_{t}\left(-\frac{1}{2}\left|\nabla Q_{s} f\right|^{2}\right)+d P_{t}\left(\left|\nabla Q_{s} f\right|\right) \\
& \leq \frac{d^{2}}{2} .
\end{aligned}
$$

Hence

$$
P_{t}\left(Q_{1} f\right)(x)-P_{t} f(y)=\phi(1)-\phi(0)=\int_{0}^{1} \phi^{\prime}(s) d s \leq \frac{d^{2}}{2}
$$

which is the result. 
(ii) Second alternate proof. This second alternate proof also uses the Hopf-Lax infimum-convolution semigroup as solution of the Hamilton-Jacobi equation, and relies on the hypercontractivity property along the heat flow recently put forward in [6]. Namely, by the log-Harnack inequality (1.7), for $f$ say bounded continuous and $v>0$,

$$
P_{t}\left(Q_{1} f\right) \leq \frac{1}{v} Q_{2 t}\left(\log P_{t}\left(\mathrm{e}^{v Q_{1} f}\right)\right) .
$$

Under non-negative curvature, it is shown in [6] that for every (bounded continuous) $\psi: M \rightarrow \mathbb{R}$ and $t>0$,

$$
\log P_{t}\left(e^{Q_{2 t} \psi}\right) \leq P_{t} \psi
$$

With $v=1 / 2 t$ and $\psi=f / 2 t$, the conclusion immediately follows by homogeneity of the infimum-convolutions.

(iii) Third alternate proof. This proof may be obtained by linear approximations of the Hamilton-Jacobi equation (vanishing viscosity method) along the lines of [12]. Following the notation therein, let for every $\varepsilon>0$, the approximated Hopf-Lax semigroup

$$
Q_{t}^{\varepsilon} f=-2 \varepsilon \log P_{\varepsilon t}\left(\mathrm{e}^{-f / 2 \varepsilon}\right)
$$

solution of the equation

$$
\partial_{t} u=\varepsilon \mathrm{L} u-\frac{1}{2}|\nabla u|^{2} .
$$

In a sense which can be made precise, $\lim _{\varepsilon \rightarrow 0} Q_{t}^{\varepsilon} f=Q_{t} f$. Dealing with

$$
\phi(s)=P_{s}\left(Q_{1}^{\varepsilon}\left(P_{t-s} f\right)\right), \quad s \in[0, t],
$$

shows that

$$
\phi^{\prime}(s)=2 \varepsilon P_{s}\left(\frac{1}{P_{\varepsilon} g}\left[\frac{\left|\nabla P_{\varepsilon} g\right|^{2}}{P_{\varepsilon} g}-P_{\varepsilon}\left(\frac{|\nabla g|^{2}}{g}\right)\right]\right)
$$

where $g=\mathrm{e}^{-P_{t-s} f / 2 \varepsilon}$. Under the gradient bound (1.6), $\phi^{\prime}(s) \leq 0$ which yields that

$$
P_{t}\left(Q_{1}^{\varepsilon} f\right) \leq Q_{1}^{\varepsilon}\left(P_{t} f\right) .
$$

In the limit as $\varepsilon \rightarrow 0$, the announced commutation property follows.

One benefit of the third alternate proof is that it may be developed similarly on the curvature-dimension condition $C D(0, N)$ with a finite-dimensional parameter $N$, for example with $N=n$ for the Laplace operator on an $n$-dimensional Riemannian manifold with non-negative Ricci curvature ( $c f . \quad[5,8])$. We sketch the argument. The local logarithmic Sobolev inequalities of [10] (see also [8]) under $C D(0, N)$ ensure after linearization that, for any $t>0$, any non-negative smooth function $g: M \rightarrow \mathbb{R}$ and any $c>0$,

$$
c \frac{\left|\nabla P_{t} g\right|^{2}}{P_{t} g}-P_{t}\left(\frac{|\nabla g|^{2}}{g}\right) \leq(c-1) P_{t}(\mathrm{~L} g)+\frac{N}{2 t}(\sqrt{c}-1)^{2} P_{t} g .
$$


Arguing as previously in the $C D(0, \infty)$ case then yields that for any $f$ and $t, s>0$,

$$
P_{t}\left(Q_{1}^{\varepsilon} f\right) \leq Q_{1}^{\varepsilon}\left(P_{s} f\right)+N(\sqrt{t}-\sqrt{s})^{2}
$$

and similarly in the limit as $\varepsilon \rightarrow 0$.

Applied to the Wasserstein contraction, the latter shows that, under $C D(0, N)$ and in the notation of Corollary 4.2,

$$
W_{2}^{2}\left(\mu_{t}, v_{s}\right) \leq W_{2}^{2}\left(\mu_{0}, \nu_{0}\right)+2 N(\sqrt{t}-\sqrt{s})^{2} .
$$

This inequality covers (4.2), however only when $s=t$. Note that when $s=0$ and $\mu_{t}=v_{t}$, then $W_{2}\left(\mu_{t}, \mu_{0}\right) \leq \sqrt{2 N t}$ which describes a classical behavior of Brownian motion in Euclidean space. Further Wasserstein contraction properties under curvature-dimension condition are emphasized in [14,20,27,41].

It should be mentioned in addition that, following the argument at the end of Section 4, the contraction inequality (5.1) implies back the commutation

$$
P_{t}\left(Q_{1} f\right) \leq Q_{1}\left(P_{s} f\right)+N(\sqrt{t}-\sqrt{s})^{2}
$$

for all (bounded smooth) $f: M \rightarrow \mathbb{R}$ and $t, s>0$. Now, given $a \in \mathbb{R}$ to be specified, set $t=(1+\varepsilon a) s$ for $\varepsilon>0$ small enough. By homogeneity, the latter yields

$$
P_{(1+a \varepsilon) s}\left(Q_{\varepsilon} f\right) \leq Q_{\varepsilon}\left(P_{s} f\right)+\frac{N a^{2} \varepsilon s}{(\sqrt{1+a \varepsilon}+1)^{2}} .
$$

A Taylor expansion at $\varepsilon=0$ then shows that

$$
\operatorname{as}_{s}(\mathrm{~L} f)-\frac{1}{2} P_{s}\left(|\nabla f|^{2}\right) \leq-\frac{1}{2}\left|\nabla P_{s} f\right|^{2}+\frac{N a^{2} s}{4} \text {. }
$$

For $a=\frac{2}{N} \mathrm{~L} P_{s} f$, it follows that

$$
\left|\nabla P_{s} f\right|^{2} \leq P_{s}\left(|\nabla f|^{2}\right)-\frac{2 s}{N}\left(\mathrm{~L} P_{s} f\right)^{2} .
$$

This inequality, holding (pointwise) for every (smooth) $f$ and every $s>0$, is known to be equivalent to the curvature-dimension condition $C D(0, N)(c f .((1.11)$ in [10], or [41]). As such, the Wasserstein contraction (5.1), as well as the dimensional commutation (5.2), are also equivalent to $C D(0, N)$.

\section{Links with the Evolutionary Variational Inequality}

To conclude this work, we briefly describe some of the connections between the preceding material and recent contributions around the so-called Evolutionary Variational Inequality (EVI). As mentioned in the introduction, the EVI has been recently 
developed by L. Ambrosio, N. Gigli and G. Savaré [2-4] towards the connection between the curvature condition $C D(K, \infty)$ in the sense of the $\Gamma_{2}$ operator of [7] (see $[5,8]$ ), expressed here through the commutation (1.6), and the curvature bound in the sense of Lott-Villani-Sturm in metric measure spaces as convexity of relative entropy along the geodesics of optimal transportation [30,35,37]. The recent contribution [20] addresses corresponding issues for the curvature-dimension condition $C D(K, N)$ (in particular in connection with (5.3))

The purpose of this short paragraph is to describe the idea at the root of the EVI following the recent main development [4]. In this work, the authors actually establish the EVI in the extended class of Riemannian energy measure spaces, providing a complete link between the Bakry-Émery $\Gamma_{2}$ and Lott-Villani-Sturm curvatures (the implication from Lott-Villani-Sturm to $\Gamma_{2}$ was achieved in $[2,3]$ ). With respect to [4], we only concentrate here on the main principle of proof in the simplified framework of weighted Riemannian manifolds, the main achievement of [4] being actually to perform the argument in a much larger class of non-smooth spaces together with a rather involved analysis. The guideline of this investigation is the Eulerian approach of [33] and [17] but the non-smooth structure causes a lot of technical problems. We nevertheless found it useful to outline the argument, avoiding all the regularity issues, in the context of this paper to illustrate the general principle and the links with the material of the previous sections. We of course refer to [4] (and $[2,3]$ ) for a complete rigorous investigation.

For simplicity thus, we deal with the weighted Riemannian framework of the preceding sections with $d \mu=\mathrm{e}^{-V} d x$ a probability measure, and restrict ourselves to the non-negative curvature assumption $C D(0, \infty)$ expressed in the form of the commutation property (1.6) with $K=0$. The case of arbitrary $K \in \mathbb{R}$ is easily adapted along the same lines $c f$. [4].

Let $f$ and $g$ be probability densities with respect to the probability measure $\mu$. The Evolutionary Variational Inequality (EVI) indicates that under $C D(0, \infty)$, for any $t>0$,

$$
\mathrm{W}_{2}^{2}\left(\mu_{t}, \nu_{0}\right)+2 t \int_{M} P_{t} f \log P_{t} f d \mu \leq \mathrm{W}_{2}^{2}\left(\mu_{0}, \nu_{0}\right)+2 t \int_{M} g \log g d \mu
$$

where $d \mu_{t}=P_{t} f d \mu, d v_{t}=P_{t} g d \mu$. In the limit as $t \rightarrow 0$, together with the semigroup property,

$$
\frac{1}{2} \frac{d}{d t} \mathrm{~W}_{2}^{2}\left(\mu_{t}, v_{0}\right) \leq \int_{M} g \log g d \mu-\int_{M} P_{t} f \log P_{t} f d \mu
$$

(the derivative being understood in an extended sense as the limsup of the right difference quotient).

The material described in the preceding sections gets close to (6.1), however not quite. Indeed, the conjunction of (1.11) and of the Wasserstein contraction (4.2) (for $K=0$ ) yields

$$
\mathrm{W}_{2}^{2}\left(\mu_{t}, v_{t}\right)+2 t \int_{M} P_{t} f \log P_{t} f d \mu \leq \frac{3}{2} \mathrm{~W}_{2}^{2}\left(\mu_{0}, v_{0}\right)+2 t \int_{M} g \log g d \mu
$$


which is not directly comparable to (6.1) but which, in any case, is useless in the limit as $t \rightarrow 0$. To reach EVI, more on optimal transportation is actually required.

One key step in this regard is the existence of curves of probability densities $h_{s}, s \in[0,1]$, with respect to $\mu$ interpolating between $h_{0}=g$ and $h_{1}=f$, assumed to be smooth both in space and $s$, such that for every smooth function $\psi$ on $M$,

$$
\int_{M} \dot{h}_{s} \psi d \mu \leq \frac{1}{2} \mathrm{~W}_{2}^{2}\left(\mu_{0}, \nu_{0}\right)+\frac{1}{2} \int_{M}|\nabla \psi|^{2} h_{s} d \mu .
$$

Such curves are naturally provided by optimal transportation, and arise for example in the Benamou-Brenier dynamical description of the Wasserstein distance [11] (cf. [1,36,37]). Actually, the existence of geodesics $\mu_{s}=h_{s} \mu$ in the Wasserstein space satifying (6.3) just depends on the length property of the space and is a general result of [28]. In general, it is however not even clear that such a curve $\mu_{s}$ is absolutely continuous with respect to $\mu$, so the basic issue here concerns regularity of $\mu_{s}$ and $h_{s}$. Even in a smooth context, there is an correction error in (6.3) which may be shown to be negligible for the further purposes so that for simplicity we ignore it here. The existence and regularity of such curves $h_{s}, s \in[0,1]$, satisfying (6.3) in a non-smooth setting is a delicate issue carefully investigated in [4].

To illustrate at a mild level such curves, and in $M=\mathbb{R}^{n}$ for the simplicity of the notation (the manifold case being similar at the expense of further Riemannian technology, $c f$. [37]), consider the Brenier map $T: \mathbb{R}^{n} \rightarrow \mathbb{R}^{n}$ pushing forward $d \mu_{0}=f d \mu$ to $d \nu_{0}=g d \mu$ and providing optimal transportation in the sense of the Wasserstein distance $W_{2}$ as

$$
\mathrm{W}_{2}^{2}\left(\mu_{0}, v_{0}\right)=\int_{\mathbb{R}^{n}}|x-T(x)|^{2} f(x) d \mu(x)
$$

(cf. $[1,36,37] \ldots)$. Consider then the geodesics $T_{s}=s \mathrm{Id}+(1-s) T, s \in[0,1]$, of optimal transportation. If $h_{s}$ denotes the density with respect to $\mu$ of the pushforward measure of $d \mu_{0}=f d \mu$ by $T_{s}$ (so that $h_{0}=g$ and $h_{1}=f$ ) assumed to be smooth both in space and $s$, it is easily checked that for every smooth function $\psi$ on $\mathbb{R}^{n}$,

$$
\int_{\mathbb{R}^{n}} \dot{h}_{s} \psi d \mu=\int_{\mathbb{R}^{n}}(x-T(x)) \cdot \nabla \psi\left(T_{s}(x)\right) f(x) d \mu(x)
$$

yielding (6.3) by the quadratic inequality and (6.4).

On the basis of (6.3), the EVI (6.1) may be analyzed by a suitable coupling between the heat kernel and optimal transportation parametrizations. Precisely, the expressions

$$
\int_{M} Q_{1} \varphi P_{t} f d \mu-\int_{M} \varphi g d \mu+t\left(\int_{M} P_{t} f \log P_{t} f d \mu-\int_{M} g \log g d \mu\right)
$$

for any smooth $\varphi$ on $M$ may be represented as

$$
\int_{0}^{1}\left(\frac{d}{d s} \int_{M} Q_{s} \varphi P_{s t} h_{s} d \mu+t \frac{d}{d s} \int_{M} P_{s t} h_{s} \log P_{s t} h_{s} d \mu\right) d s .
$$


Now, again under suitable smoothness assumptions not detailed here, by the Hamilton-Jacobi equation and integration by parts,

$$
\begin{aligned}
\frac{d}{d s} \int_{M} Q_{s} \varphi P_{s t} h_{s} d \mu= & -\frac{1}{2} \int_{M}\left|\nabla Q_{s} \varphi\right|^{2} P_{s t} h_{s} d \mu+\int_{M} \dot{h}_{s} P_{s t}\left(Q_{s} \varphi\right) d \mu \\
& +t \int_{M} Q_{s} \varphi \mathrm{L} P_{s t} h_{s} d \mu \\
= & -\frac{1}{2} \int_{M}\left|\nabla Q_{s} \varphi\right|^{2} P_{s t} h_{s} d \mu+\int_{M} \dot{h}_{s} P_{s t}\left(Q_{s} \varphi\right) d \mu \\
& -t \int_{M} \nabla Q_{s} \varphi \cdot \nabla P_{s t} h_{s} d \mu .
\end{aligned}
$$

On the other hand,

$$
\begin{aligned}
\frac{d}{d s} \int_{M} P_{s t} h_{s} \log P_{s t} h_{s} d \mu & =\int_{M}\left[P_{s t} \dot{h}_{s}+t \mathrm{~L} P_{s t} h_{s}\right] \log P_{s t} h_{s} d \mu \\
& =\int_{M} P_{s t} \dot{h}_{s} \log P_{s t} h_{s} d \mu-t \int_{M} \nabla P_{s t} h_{s} \cdot \nabla\left(\log P_{s t} h_{s}\right) d \mu
\end{aligned}
$$

where we used that $\frac{d}{d s} P_{s t} h_{s}=P_{s t} \dot{h}_{s}+t \mathrm{~L} P_{s t} h_{s}$ and $\int_{M} P_{s t} \dot{h}_{s} d \mu=\int_{M} \dot{h}_{s} d \mu=0$.

From these expressions, it is easily checked that the sum

$$
\frac{d}{d s} \int_{M} Q_{s} \varphi P_{s t} h_{s} d \mu+t \frac{d}{d s} \int_{M} P_{s t} h_{s} \log P_{s t} h_{s} d \mu
$$

may be rearranged as

$$
\begin{aligned}
-\frac{1}{2} \int_{M} \mid \nabla\left(Q_{s} \varphi\right. & \left.+t \log P_{s t} h_{s}\right)\left.\right|^{2} P_{s t} h_{s} d \mu-\frac{t^{2}}{2} \int_{M} \frac{\left|\nabla P_{s t} h_{s}\right|^{2}}{P_{s t} h_{s}} d \mu \\
& +\int_{M} \dot{h}_{s} P_{s t}\left(Q_{s} \varphi+t \log P_{s t} h_{s}\right) d \mu .
\end{aligned}
$$

Forgetting the term $\frac{t^{2}}{2} \int_{M} \frac{\left|\nabla P_{s t} h_{s}\right|^{2}}{P_{s t} h_{s}} d \mu$ (which is anyway of the order $o(t)$ in the limit (6.2)), this quantity is upper-bounded by

$$
-\frac{1}{2} \int_{M} P_{s t}\left(\left|\nabla\left(Q_{s} \varphi+t \log P_{s t} h_{s}\right)\right|^{2}\right) h_{s} d \mu+\int_{M} \dot{h}_{s} P_{s t}\left(Q_{s} \varphi+t \log P_{s t} h_{s}\right) d \mu
$$

where we used symmetry of the semigroup. Now, by the curvature condition in the form of the commutation (1.6), the latter is further upper-bounded by

$$
-\frac{1}{2} \int_{M}\left|\nabla P_{s t}\left(Q_{s} \varphi+t \log P_{s t} h_{s}\right)\right|^{2} h_{s} d \mu+\int_{M} \dot{h}_{s} P_{s t}\left(Q_{s} \varphi+t \log P_{s t} h_{s}\right) d \mu .
$$


With $\psi=P_{s t}\left(Q_{s} \varphi+t \log P_{s t} h_{s}\right)$, (6.3) implies that this expression is precisely bounded from above by $\mathrm{W}_{2}^{2}\left(\mu_{0}, v_{0}\right)$. Integrating in $s$ from 0 to 1 and taking the supremum over all $\varphi$ 's then yields the announced EVI (6.1). It might be worthwhile mentioning that with respect to (1.6) only the weaker commutation property $\left|\nabla P_{t} g\right|^{2} \leq \mathrm{e}^{-K t} P_{t}\left(|\nabla g|^{2}\right)$ is used here.

As mentioned above, the preceding argument is inspired by the Eulerian calculus developed by F. Otto and M. Westdickenberg [33] in their approach of the contraction property (4.2). Namely, if the parametrization does not involve the heat flow, consider for $\varphi: M \rightarrow \mathbb{R}$ smooth enough,

$$
\int_{M} Q_{1} \varphi P_{t} f d \mu-\int_{M} \varphi P_{t} g d \mu=\int_{0}^{1}\left(\frac{d}{d s} \int_{M} Q_{s} \varphi P_{t} h_{s} d \mu\right) d s .
$$

Since, as above,

$$
\frac{d}{d s} \int_{M} Q_{s} \varphi P_{t} h_{s} d \mu=-\frac{1}{2} \int_{M}\left|\nabla Q_{s} \varphi\right|^{2} P_{t} h_{s} d \mu+\int_{M} \dot{h}_{s} P_{t}\left(Q_{s} \varphi\right) d \mu,
$$

by time reversibility and the gradient bound (1.6),

$$
\begin{aligned}
\frac{d}{d s} \int_{M} Q_{s} \varphi P_{t} h_{s} d \mu & =-\frac{1}{2} \int_{M} P_{t}\left(\left|\nabla Q_{s} \varphi\right|^{2}\right) h_{s} d \mu+\int_{M} \dot{h}_{s} P_{t}\left(Q_{s} \varphi\right) d \mu \\
& \leq-\frac{1}{2} \int_{M}\left|\nabla P_{t}\left(Q_{s} \varphi\right)\right|^{2} h_{s} d \mu+\int_{M} \dot{h}_{s} P_{t}\left(Q_{s} \varphi\right) d \mu .
\end{aligned}
$$

Using (6.3) then yields

$$
\int_{M} Q_{1} \varphi P_{t} f d \mu-\int_{M} \varphi P_{t} g d \mu \leq \frac{1}{2} W_{2}^{2}\left(\mu_{0}, \nu_{0}\right),
$$

that is, after taking the supremum in $\varphi$, the contraction property (4.2) of Corollary 4.2 .

\section{References}

[1] L. Ambrosio, N. Gigli and G. Savaré, "Gradient Flows in Metric Spaces and in the Space of Probability Measures", Lectures in Mathematics ETH, Birkhäuser, Zürich, 2008.

[2] L. Ambrosio, N. Gigli and G. SaVAré, Calculus and heat flow in metric measure spaces and applications to spaces with Ricci bounds from below, Invent. Math. 195 (2014), 289-391.

[3] L. Ambrosio, N. Gigli and G. Savaré, Metric measures spaces with Riemannian Ricci curvature bounded from below, Duke Math. J. 163 (2014), 1405-1490.

[4] L. Ambrosio, N. Gigli and G. SaVAré, Bakry-Émery curvature-dimension condition and Riemannian Ricci curvature bounds, Ann. Probab. 43 (2015), 339-404.

[5] D. BAKRY, L'hypercontractivité et son utilisation en théorie des semigroupes, Ecole d'Eté de Probabilités de Saint-Flour, Lecture Notes in Math., Vol. 1581, 1994, 1-114. 
[6] D. BAKRy, F. Bolley and I. Gentil, Dimension dependent hypercontractivity for Gaussian kernels, Probab. Theory Related Fields 154 (2012), 845-874.

[7] D. BAKRY and M. ÉMERY, Diffusions hypercontractives, In: "Séminaire de Probabilités" XIX, 1983/84, Lecture Notes in Math., Vol. 1123, 1985, 177-206.

[8] D. BAKRY, I. GenTIL and M. LedouX, "Analysis and Geometry of Markov Diffusion Operators", Grundlehren der Mathematischen Wissenschaften, Vol. 348, Springer, 2014.

[9] D. BAKRY and M. LEDOUX, Lévy-Gromov's isoperimetric inequality for an infinitedimensional diffusion generator, Invent. Math. 123 (1996), 259-281.

[10] D. BAKRY and M. LEDOUX, A logarithmic Sobolev form of the Li-Yau parabolic inequality, Rev. Mat. Iberoam. 22 (2006), 683-702.

[11] J.-D. BENAMOU and Y. BRENIER, A computional fluid mechanics solution to the MongeKantorovich mass transfer problem, Numer. Math. 84 (2000), 375-393.

[12] S. BobKov, I. Gentil and M. Ledoux, Hypercontractivity of Hamilton-Jacobi equations, J. Math. Pures Appl. 80 (2001), 669-696.

[13] S. BobKOV and C. Houdré, Some connections between isoperimetric and Sobolev-type inequalities, Mem. Amer. Math. Soc., Vol. 129, 1997.

[14] F. Bolley, I. Gentil and A. Guillin, Dimensional contraction via Markov transportation distance, J. Lond. Math. Soc. (2) 90 (2014), 309-332.

[15] J. CARRILLO, R. MCCANN and C. VILLANI, Kinetic equilibration rates for granular media and related equations: entropy dissipation and mass transportation estimates, Rev. Mat. Iberoam. 19 (2003), 971-1018.

[16] D. CORDERO-ERAUSQUIN, Some applications of mass transport to Gaussian-type inequalities, Arch. Ration. Mech. Anal. 161 (2002), 257-269.

[17] S. DANERI and G. S AVARÉ, Eulerian calculus for the displacement convexity in the Wasserstein distance, SIAM J. Math. Anal. 40 (2008), 1104-1122.

[18] E. B. DAVIES, "Heat Kernels and Spectral Theory", Cambridge Tracts in Mathematics 92, Cambridge, 1989.

[19] M. ERBAR, The heat equation on manifolds as a gradient flow in the Wasserstein space, Ann. Inst. Henri Poincaré Probab. Stat. 46 (2010), 1-23.

[20] M. ERBAR, K. KUWADA and K.-T. STURM, On the equivalence of the entropic curvaturedimension condition and Bochner's inequality on metric measure spaces, Invent. Math. 201 (2015), 993-1071.

[21] L. Evans, "Partial Differential Equations", Graduate Studies in Mathematics 19, American Mathematical Society, 1998.

[22] N. Gigli, K. KuwAda and S.-I. OHTA, Heat flow on Alexandrov spaces, Comm. Pure Appl. Math. 66 (2013), 307-331.

[23] N. Gigli and M. Ledoux, From log Sobolev to Talagrand: a quick proof, Discrete Cont. Dyn. Syst. 33 (2013), 1927-1935.

[24] M. Hino, On short time asymptotic behavior of some symmetric diffusions on general state spaces, Potential Anal. 16 (2002), 249-264.

[25] R. JORDAN, D. KINDERLEHRER and F. OTTO, The variational formulation of the FokkerPlanck equation, SIAM J. Math. Anal. 29 (1998), 1-17.

[26] K. KuWADA, Duality on gradient estimates and Wasserstein controls, J. Funct. Anal. 258 (2010), 3758-3774.

[27] K. KuWADA, Space-time Wasserstein controls and Bakry-Ledoux type gradient estimates, Calc. Var. Partial Differential Equations, to appear.

[28] S. Lisini, Characterization of absolutely continuous curves in Wasserstein spaces, Calc. Var. Partial Differential Equations 28 (2007), 85-120.

[29] P. LI and S.-T. YAU, On the parabolic kernel of the Schrödinger operator, Acta Math. 156 (1986), 153-201.

[30] J. LOTT and C. VILLANI, Ricci curvature for metric-measure spaces via optimal transport, Ann. of Math. 169 (2009), 903-991. 
[31] F. Отто, The geometry of dissipative evolution equations: the porous medium equation, Comm. Partial Differential Equations 26 (2001), 101-174.

[32] F. OTTO and C. VILlani, Generalization of an inequality by Talagrand, and links with the logarithmic Sobolev inequality, J. Funct. Anal. 173 (2000), 361-400.

[33] F. OTTO and M. WeSTDICKENBERG, Eulerian calculus for the contraction in the Wasserstein distance, SIAM J. Math. Anal. 37 (2005), 1227-1255.

[34] M.-K. VON RENESSE and K.-T. STURM, Transport inequalities, gradient estimates, entropy and Ricci Curvature, Comm. Pure Appl. Math. 68 (2005), 923-940.

[35] K.-T. STURM, On the geometry of metric measure spaces I \& II, Acta Math. 196 (2006), 65-131, 133-177.

[36] C. VILlani, "Topics in Optimal Transportation", Graduate Studies in Mathematics, Vol. 58, American Mathematical Society, 2003.

[37] C. Villani, "Optimal Transport, Old and New", Grundlehren der Mathematischen Wissenschaften, Vol. 338, Springer, 2009.

[38] F.-Y. WANG, Logarithmic Sobolev inequalities on noncompact Riemannian manifolds, Probab. Theory Related Fields 109 (1997), 417-424.

[39] F.-Y. WANG, "Functional Inequalities, Markov Properties and Spectral Theory", Science Press, 2005.

[40] F.-Y. WANG, Harnack inequalities on manifolds with boundary and applications, J. Math. Pures Appl. 94 (2010), 304-321.

[41] F.-Y. WANG, Equivalent semigroup properties for the curvature-dimension condition, Bull. Sci. Math. 135 (2011), 803-815.

Institut de Mathématiques de Toulouse Université de Toulouse - Paul Sebatier 31062 Toulouse, France bakry@math.univ-toulouse.fr ledoux@math.univ-toulouse.fr

Institut Camille Jordan UMR CNRS 5208

Université Claude Bernard Lyon 1 69622 Lyon, France gentil@math.univ-lyon1.fr 